\title{
Effectiveness and Safety of the Combination of the Traditional Chinese Medicine Prescription Jade Screen and Desloratadine in the Treatment of Chronic Urticaria: A Systematic Review and Meta-Analysis of Randomized Controlled Trials
}

\author{
Shao-fei Yuan, ${ }^{1}$ Jing-zhi Guan, ${ }^{2}$ Yong Hao, ${ }^{3}$ Jian-yuan Chai, ${ }^{4}$ Feng-li Gao, ${ }^{1}$ Su-qin Shi, \\ Jian-xin Wang, ${ }^{5} \mathrm{Jin}_{\mathrm{He}}{ }^{6}$ and Ji-hai Shi ${ }^{3}$ \\ ${ }^{1}$ Pharmaceutical Department, The Second Affiliated Hospital of Baotou Medical College, No. 30 Hudemulin Road, Baotou, \\ Inner Mongolia 014030, China \\ ${ }^{2}$ Pharmaceutical Department, Inner Mongolia International Mongolian Hospital, No. 83 Daxue East Road, Hohhot, \\ Inner Mongolia 010065, China \\ ${ }^{3}$ Dermatological Department, The Second Affiliated Hospital of Baotou Medical College, No. 30 Hudemulin Road, Baotou, \\ Inner Mongolia 014030, China \\ ${ }^{4}$ Department of Gastroenterology, The Second Affiliated Hospital of Baotou Medical College, No. 30 Hudemulin Road, Baotou, \\ Inner Mongolia 014030, China \\ ${ }^{5}$ Human Anatomy and Tissue Embryology, Weifang Medical University, No. 7166 Baotong Street, Weicheng District, \\ Weifang, Shandong 261021, China \\ ${ }^{6}$ Development Department, Inner Mongolia International Mongolian Hospital, No. 83 Daxue East Road, Hohhot, \\ Inner Mongolia 010065, China
}

Correspondence should be addressed to Ji-hai Shi; jihaishi_imimh@163.com

Received 15 June 2017; Revised 3 October 2017; Accepted 17 October 2017; Published 12 November 2017

Academic Editor: Subash C. Gupta

Copyright (C) 2017 Shao-fei Yuan et al. This is an open access article distributed under the Creative Commons Attribution License, which permits unrestricted use, distribution, and reproduction in any medium, provided the original work is properly cited.

Objective. The aim of this study is to systematically evaluate the clinical efficacy and safety of the traditional Chinese medicine prescription Jade Screen combined with desloratadine in the treatment of chronic urticaria. Methods. Two researchers independently conducted literature searches. The extracted data were analyzed using Rev Man 5.2.3 software. The established retrieval time range of the various databases was up to $15 \mathrm{March}$, 2017. Results. Sixteen randomized controlled trials were included in this study. The results of the meta-analysis showed that the total effective rate of using Jade Screen and desloratadine in combination to treat chronic urticaria was higher than that with desloratadine alone $(P<0.00001)$, while its recurrence rate $(P<0.00001)$ and symptom score $(P=0.006)$ were both significantly lower than the latter. The rate of adverse reaction in the combination group was lower than that when orally taking desloratadine alone $(P=0.74)$, and the serum level of total IgE in the combination group was lower than that when orally taking desloratadine alone $(P=0.82)$; however, the results of the rate of adverse reaction and the serum level of total IgE were insignificant. Conclusion. Using Jade Screen and desloratadine together to treat chronic urticaria gains a better clinical effect than using desloratadine alone.

\section{Introduction}

Urticaria is a common disease in dermatology, characterized by transient, localized, edematous wheals of various sizes on skin mucous membranes and accompanied by severe itching. If wheals occur every day or almost every day for more than six weeks, such condition is called chronic urticaria [1]. Approximately $0.5 \% \sim 1.0 \%$ of the world population has chronic urticaria [2]. With the aggravation of urban pollution and the worsening of the environment, the incidence rate of urticaria is on the rise year by year. Since its occurrence shows no apparent pattern, the disease recurs easily, and the 
health index of the patients $[3,4]$ and their quality of life decline $[5,6]$. The conventional treatment method involves the single use or combined use of antihistamine drugs [7]. However, these drugs can only relieve the symptoms of some patients, and the overall effect is not significant. The disease easily relapses after withdrawal [8]. Thus, there is an urgent need to seek new therapeutic strategies [2].

Urticaria is called "hidden rash" or "red and white wandering wind" in traditional Chinese medicine. As the "Treatise on Needling" in the Sù Wèn ("Plain Questions") states, when shaoyin qi is excessive, skin numbness and hidden rash will occur, which is also recorded in 《General Treatise on Causes and Manifestations of All Diseases》, 《Invaluable Prescriptions for Ready Reference》, and 《Complete Works of Diagnosis and Treatment for Surgical Diseases and other works》. Most modern doctors believe that rheumatism attacks the skin but decreased resistance and transportation dysfunction of the spleen and stomach are the keys to the disease [9]. It can be seen that strengthening the spleen and nourishing qi can not only expel the invasive wind-pathogen out of the body but also prevent it from spreading within the body. Traditional Chinese medicines strengthening the spleen, nourishing qi, dispelling wind, and arresting itching have the functions to improve the immune system and to eliminate pathogens, thereby controlling the cause and manifestation of chronic urticaria. Jade Screen is a classical prescription that comes from Danxi's Mastery of Medicine by Zhu Dan-xi, a great master of the Yuan Dynasty. As a basic Chinese medicine, this prescription has a good therapeutic effect. It has been listed into Chinese Pharmacopoeia [10]. Jade Screen formula consists of three traditional Chinese medicines, including Astragalus membranaceus [Astragalus membranaceus (Fisch.) Bunge]; Rhizoma Atractylodis Macrocephalae (bighead atractylodes rhizome); and Radix Saposhnikoviae [Saposhnikovia divaricata (Turcz.) Schischk]. Astragalus membranaceus can nourish qi and strengthen the exterior to enhance the grain of the skin and the texture of the subcutaneous flesh and prevent it from evil invasion; Rhizoma Atractylodis Macrocephalae strengthens the middle warmer and invigorates the spleen to recover vital qi, and Radix Saposhnikoviae dispels wind and disperses evil to prevent external invasion and help cure various diseases. The compatibility of the three medicines has the functions of nourishing qi, strengthening the exterior, and arresting sweat. With sweet flavor and warm nature, Astragalus membranaceus tonifies qi and strengthens the exterior, while Rhizoma Atractylodis Macrocephalae invigorates the spleen and eliminates dampness. The combination of Astragalus membranaceus and Rhizoma Atractylodis Macrocephalae can greatly tonify lung and stomach qi, make the spleen and stomach healthy and vigorous, replenish the fleshy exterior, and prevent evil invasion, while Radix Saposhnikoviae relieves the exterior syndrome by dispersing wind-evil.

Among published clinical studies, some reports argue that Jade Screen combined with desloratadine can achieve a good therapeutic effect on chronic urticaria. To comprehensively assess the clinical efficacy and safety of this classical ancient prescription, we conducted this systematic review.

\section{Methods and Analysis}

2.1. Search Strategy. Records from the following databases in Chinese or English have been retrieved up to 15 March, 2017: Cochrane Library, PubMed, Embase, SinoMed, CNKI, VIP, and WanFang Data. The retrieval strategy was based on the combination of subject terms and free words. Chinese search terms were "Jade Screen", "dilvleitading", "xunmazhen", etc.; English search terms were "Yu Ping Feng", "Jade Screen", "traditional Chinese medicine", "Urticaria", "Urticarias", "desloratadine", "Clarinex" and so on. Manual retrieval was conducted in 《Lishizhen Medicine and Materia Medica Research》, 《China Journal of Experimental Traditional Medical Formulae》, 《Journal of Clinical Dermatology》, 《the Chinese Journal of Dermatovenereology》, 《Liaoning Journal of Traditional Chinese Medicine $》$, and relevant conference papers and proceedings after 2010 to improve the recall ratio.

\subsection{Inclusion and Exclusion Criteria}

2.2.1. Type of Study. Jade Screen combined with desloratadine treatment of chronic urticaria in randomized controlled trials was included, including literature limited to the Chinese or English languages and regardless of whether the studies were blinded.

2.2.2. Subject Investigated. (1) Patient age and sex were recorded. The patient's region is not limited, and the diagnosis of chronic urticaria is referred to as "urticaria", 《Chinese Medical Association clinical treatment guidelines. Skin disease and STD Volume》, 《Practical Dermatology》, 《Clinical dermatology》," 《Dermatology》 [11-15]. (2) Patients who recently did not take antihistamines and glucocorticoid drugs were included.

2.2.3. Intervention. The test group is Jade Screen combined with desloratadine treatment; the control group is desloratadine alone treatment. In both groups, treatment time and dose were not limited.

2.2.4. Outcome Indicators. (1) Total efficiency refers to EAACI/GA2LEN/EDF/WAO scoring criteria according to the severity of clinical symptoms using 4 scores; the total score is the sum of the individual scores. Symptom Score Reducing Index (SSRI) $=$ (total score before treatment total score after treatment)/total score before treatment $x$ $100 \%$. Cured means that SSRI is reduced by more than $90 \%$; markedly means that SSRI is reduced by $60 \%$ to $89 \%$; improved means that SSRI is reduced by $20 \%$ to $59 \%$; invalid means that SSRI is reduced by $20 \%$ or less. The total effective rate (number of cured cases + significant cases)/the total number of patients $\times 100 \%$; (2) recurrence rate is occurrence of healing criteria in patients with follow-up after 4 weeks to 6 months; (3) adverse reaction rate is the main symptoms which are dry mouth, nausea, headache, dizziness, fatigue, and lethargy; (4) symptom score and (5) serum total IgE levels are outcome indicators. 
2.2.5. Exclusion Criteria. The exclusion criteria included the following: (1) non-RCT literature; (2) urticaria with other diseases; (3) patients with inconsistent baseline data; (4) sample content of less than 20 literature articles; (5) case reports, reviews, animal experiments, and articles with important information reported incomplete after contacting responders; or (6) treatment measures that did not meet the preselected standard settings.

2.2.6. Data Extraction. The two researchers independently read the abstracts of the documents that were retrieved. According to the exclusion criteria, the articles with obvious nonconformity were excluded, and the articles remaining that met the inclusion criteria were obtained. Further, for the noncompliant documents, a form was used to indicate the reasons for the exclusion. Finally, the data of the included articles were extracted, including title, source, author, test design, research object, research method, intervention measures, outcome measurement and evaluation, statistical analysis, recurrence, adverse reaction report, and conclusion. The two researchers cross-checked their respective average results, and if there were differences, they decided whether or not the article was included by discussing or asking the third evaluator to evaluate the article.

2.3. Quality Evaluation. According to the bias risk assessment tool recommended by the Cochrane Review Handbook 5.2, there are six aspects: (1) random allocation method; (2) allocation scheme hidden; (3) whether the survey object, treatment plan implementer, or result measure was blinded; (4) the completeness of the resulting data; (5) selective reporting of findings; and (6) other bias sources. The two reviewers independently read the full text and conducted a quality evaluation and then cross-checked the results of the quality evaluation of the trials that were difficult to determine by discussion or by the third evaluator.

2.4. Sensitivity Analysis. To study the impact of individual studies on heterogeneity, when the heterogeneity was found, if the study was deleted and the heterogeneity was significantly reduced, the study was considered as the main source of heterogeneity; the study was then further read and evaluated.

2.5. Data Analysis. Meta-analysis was performed using RevMan5.3 software. In this study, the odds ratio (OR) and its 95\% CI were used as the effect analysis statistic (MD) and its 95\% CI analysis; if the same variables were not consistent, then the mean difference (WMD) and 95\% CI analysis were used. The heterogeneity of the included studies was analyzed using the $\chi^{2}$ test (the test level was set to $\alpha=0.1$ ), and the size of the heterogeneity was determined quantitatively with $I^{2}\left(P>0.1, I^{2}>50 \%\right)$, and the results were statistically heterogeneous $\left(P \leq 0.1, I^{2}>50 \%\right)$, and the statistical heterogeneity was statistically significant $\left(P>0.1, I^{2}>\right.$ $50 \%)$. After the exclusion of significant clinical heterogeneity, the random effects model was used for the meta-analysis. Significant clinical heterogeneity was analyzed by subgroup analysis or sensitivity analysis, or descriptive analysis was performed.

\section{Results}

3.1. Retrieval Results. A total of 190 relevant publications were found using the data collection method in accordance with the retrieval strategy, including 1 from the Cochrane Library, 0 from PubMed, 3 from Embase, 34 from SinoMed, 69 from CNKI, 57 from WanFang Data, and 26 from VIP. Among them, 116 papers were obtained after duplicate checking via literature management software, and 100 articles were excluded due to the failure to meet our inclusion criteria. Finally, 16 RCTs [16-31] involving 1763 patients were included. Figure 1 shows the process and results of the literature screening.

3.2. Research Characteristics. There were 16 RCT studies that met our criteria and included in the study eventually [16-31]. These studies were published from 2008 to 2016 with a total of 1763 patients involved, including 901 in the experimental group and 862 in the control group. They were single center studies done in China. The age of the participants was from 12 to 75 , and the course of treatment ranged from 3 weeks to 17 years, Table 1.

3.3. Summary of the Quality and Bias Risk of the Trials Included. Among the 16 studies included, only 4 contained a random number table, and the rest failed to describe a random method, hidden group, blinding method, estimator's blinding method, loss to follow-up, and selection bias, Figures 2 and 3 .

3.4. Outcome Measures. All 16 studies reported their total effective rate [16-31], but only some of them included other measurements; for instance, 8 reported their recurrence rates $[19,20,22-24,27,30,31], 7$ described their rates of adverse reaction $[19,24,25,28-31], 7$ mentioned their symptom scores, 7 reported their serum total IgE levels [27, 29], and 2 reported their serum total IgE level.

3.4.1. Total Effective Rate. Among the 16 studies included [16-31], there was no heterogeneity found $\left(P=0.57, I^{2}=\right.$ $0 \%)$. Using a fixed effects model, meta-analysis indicated that the overall response rate of Jade Screen combined with desloratadine for the treatment of chronic urticaria was significantly higher than that of the oral use of desloratadine alone $[n=16, \mathrm{OR}=2.72,95 \%$ CI $(2.13,3.48), Z=7.97$, $P<0.00001]$, Figure 4 .

3.4.2. Recurrence Rate. This method [19, 20, 22-24, 27, 29, 31] was employed in 8 studies, involving 532 cases with 300 in the experimental group and 232 in the control group. Heterogeneity was still not found $\left(P=0.37, I^{2}=8 \%\right)$. The fixed effects model revealed that the recurrence rate of Jade Screen combined with desloratadine for the treatment of chronic urticaria was significantly lower than that of the oral use of desloratadine alone $[n=8, \mathrm{OR}=0.16,95 \% \mathrm{CI}$ (0.11, 0.25), $Z=8.25, P<0.00001]$, Figure 5 . 


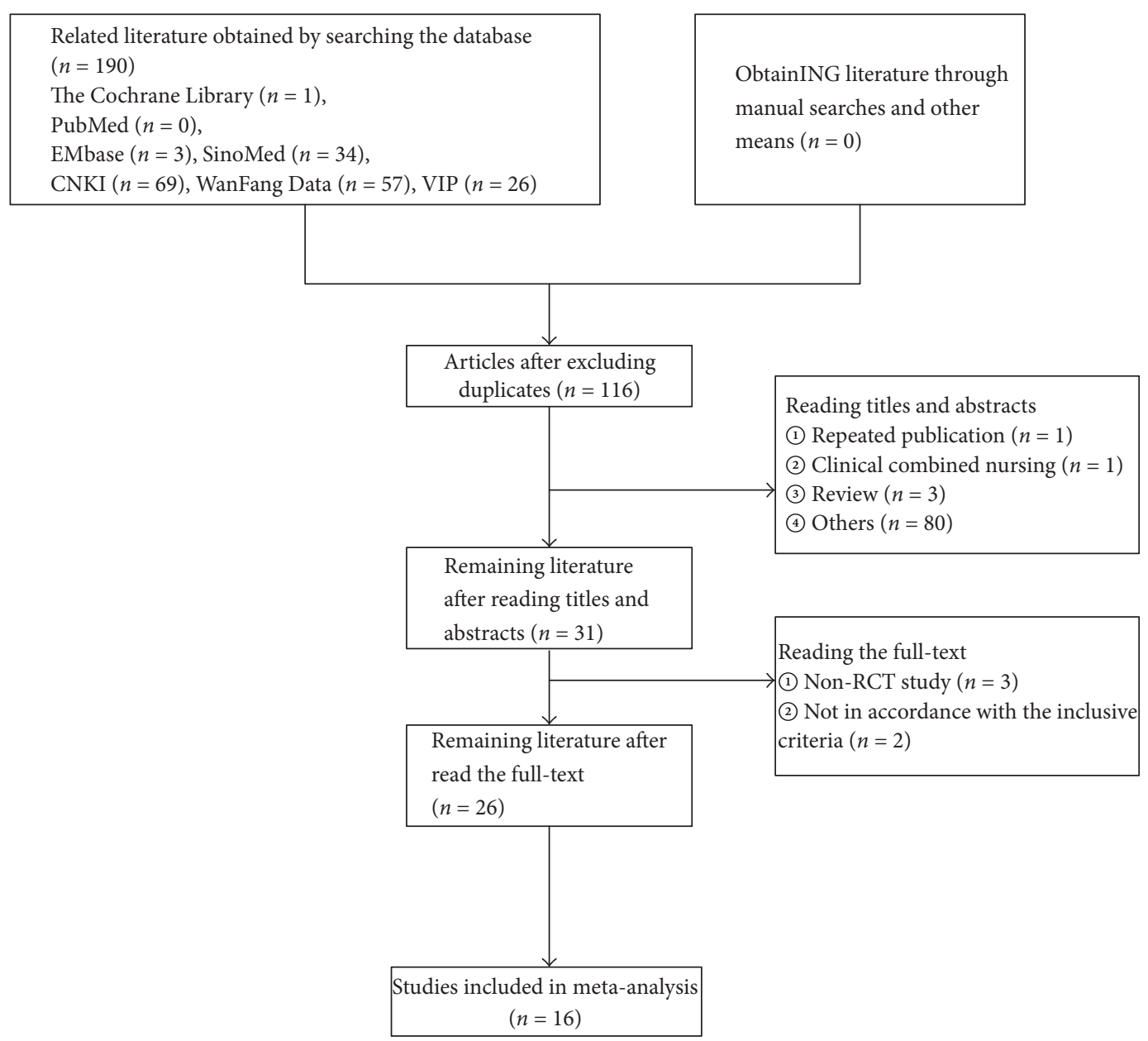

FIGURE 1: Flow diagram of the literature selection for this study.

3.4.3. Rate of Adverse Reaction. Seven studies adopted this method [19, 24, 25, 28-31], which involved 713 cases in total, including 370 in the experimental group and 343 in the control group. No heterogeneity was found $\left(P=0.93, I^{2}=\right.$ $0 \%)$. Meta-analysis suggested that the rate of adverse reaction of Jade Screen combined with desloratadine for the treatment of chronic urticaria was lower than that of the oral use of desloratadine alone but failed to show a statistical significance $[n=7, \mathrm{OR}=1.12,95 \% \mathrm{CI}(0.56,2.27), Z=0.33, P=0.74>$ $0.1]$, Figure 6.

3.4.4. Symptom Score. This method [17, 19, 27, 31] was employed in 4 studies involving 462 cases, including 229 in the experimental group and 233 in the control group. A significantly high heterogeneity was found $\left(P=0.04, I^{2}=\right.$ $64 \%)$. The random effects model indicated that the symptom score of Jade Screen combined with desloratadine for the treatment of chronic urticarial was significantly lower than that of the oral use of desloratadine alone $[n=4, \mathrm{MD}=-0.44$, 95\% CI $(-0.76,-0.13), Z=2.77, P=0.006]$, Figure 7 . For this measurement, we conducted sensitivity analysis, which showed that the heterogeneity was obviously decreased after deleting Jiang et al.s research (2009). Thus, we believe that Jiang et al.s research (2009) was the major heterogeneity source of the symptom score.

3.4.5. Serum Total IgE Level. This method [27, 30] was employed in 2 studies involving 382 cases, including 186 in the experimental group and 196 in the control group. A significantly high heterogeneity was identified $(P=0.00001$, $\left.I^{2}=99 \%\right)$. The random effects model meta-analysis did not show a significant change between Jade Screen combined with the desloratadine group and the oral use of desloratadine alone $[n=2, \mathrm{MD}=-5.93,95 \% \mathrm{CI}(-56.00,44.14), Z=0.23$, $P=0.82>0.1]$, Figure 8 .

3.5. Publication Bias. For the 16 research studies included, a funnel plot of overall response rate was drawn with the OR value of each paper as the $x$-coordinate and standard error $\log \mathrm{OR}$ value as the $y$-coordinate. The analysis result of the funnel plot showed no mean symmetric distribution on the two sides of the plot, suggesting some publication bias possibly due to the limited number of the published papers with negative results, Figure 9. 
TABLE 1: The basic characteristics of the 16 RCT studies.

\begin{tabular}{|c|c|c|c|c|c|c|c|}
\hline Author/year & Groups & Sample size & $\begin{array}{l}\text { Age (median or } \\
\text { mean or range) }\end{array}$ & Course of disease & $\begin{array}{c}\text { Course of } \\
\text { treatment (days) }\end{array}$ & Intervention & Outcomes \\
\hline \multirow{2}{*}{ Zhang et al., 2008 [16] } & $\mathrm{EP}$ & 50 & $19-60$ & 21.3 months & 28 & $(1)+(2)$ & \multirow{2}{*}{ (1)(2)(3) } \\
\hline & CG & 50 & $19-60$ & 22.5 months & 28 & (1) & \\
\hline \multirow{2}{*}{ Jiang et al., 2009 [17] } & $\mathrm{EP}$ & 32 & $18-52$ & 6-24 weeks & 84 & $(1)+(2)$ & \multirow{2}{*}{ (1) (2) (4) } \\
\hline & CG & 30 & $18-50$ & $6-25$ weeks & 84 & (1) & \\
\hline \multirow{2}{*}{ Hu et al., 2011 [18] } & $\mathrm{EP}$ & 28 & $21-58$ & 9 weeks-11 months & 28 & $(1)+(2)$ & \multirow{2}{*}{ (1) } \\
\hline & CG & 26 & $21-58$ & 9 weeks-11 months & 28 & (1) & \\
\hline \multirow{2}{*}{ Cai, 2012 [19] } & $\mathrm{EP}$ & 62 & $18-75$ & 2 months -10 years & 40 & $(1)+(2)$ & \multirow{2}{*}{ (1)(2)(3)(4) } \\
\hline & CG & 58 & $18-75$ & 2 months -10 years & 40 & (1) & \\
\hline \multirow{2}{*}{ Liang, 2012 [20] } & $\mathrm{EP}$ & 60 & $34 \pm 8.9$ & $1.1 \pm 0.25$ years & 28 & $(3)+(4)$ & \multirow{2}{*}{ (1)(2) (3) } \\
\hline & CG & 60 & $36 \pm 9.7$ & $1.6 \pm 0.5$ years & 28 & (3) & \\
\hline \multirow{2}{*}{ Qu, 2013 [21] } & $\mathrm{EP}$ & 43 & $12-45$ & 6 weeks-2 years & 28 & $(1)+(2)$ & \multirow{2}{*}{ (1) (2) } \\
\hline & CG & 43 & $13-43$ & 6 weeks-2 years & 28 & (1) & \\
\hline \multirow{2}{*}{ Su et al., 2013 [22] } & $\mathrm{EP}$ & 59 & $12-60$ & $\geq 6$ weeks & 28 & $(3)+(4)$ & \multirow{2}{*}{ (1) (2) (3) } \\
\hline & CG & 58 & $12-60$ & $\geq 6$ weeks & 28 & (3) & \\
\hline \multirow{2}{*}{ Wang, 2013 [23] } & $\mathrm{EP}$ & 99 & $35-60$ & 3 months-12 years & 28 & $(1)+(2)$ & \multirow{2}{*}{ (1) (2) (3) } \\
\hline & CG & 82 & $35-60$ & 3 months -12 years & 28 & (1) & \\
\hline \multirow{2}{*}{$\begin{array}{l}\text { Wei and Zhang, } 2013 \\
\text { [24] }\end{array}$} & $\mathrm{EP}$ & 41 & $34.5 \pm 10.7$ & $\geq 6$ weeks & 28 & $(3)+(2)$ & \multirow{2}{*}{ (1)(2)(3) } \\
\hline & CG & 40 & $37.2 \pm 12.9$ & $\geq 6$ weeks & 28 & (3) & \\
\hline \multirow{2}{*}{ Zhu, 2013 [25] } & $\mathrm{EP}$ & 36 & $34.60 \pm 4.20$ & 2 months -3 years & 21 & $(1)+(2)$ & \multirow{2}{*}{ (1)(2) (3) } \\
\hline & CG & 28 & $34.60 \pm 4.20$ & 2 months -3 years & 21 & (1) & \\
\hline \multirow{2}{*}{ Xie, 2014 [26] } & $\mathrm{EP}$ & 60 & $16-58$ & 2 months -8 years & 56 & $(1)+(2)$ & \multirow{2}{*}{ (1)(2) } \\
\hline & CG & 60 & $16-58$ & 2 months -8 years & 56 & (1) & \\
\hline \multirow{2}{*}{ Fu et al., 2015 [27] } & $\mathrm{EP}$ & 100 & $35.0 \pm 3.0$ & $4.1 \pm 1.6$ years & 56 & $(3)+(4)$ & \multirow{2}{*}{ (1)(2)(3)(4)(5) } \\
\hline & CG & 110 & $36.5 \pm 2.5$ & $4.4 \pm 1.3$ years & 56 & (3) & \\
\hline \multirow{2}{*}{$\mathrm{Li}, 2015$ [28] } & $\mathrm{EP}$ & 62 & $18-56$ & 2 months -3 years & 28 & $(1)+(2)$ & \multirow{2}{*}{ (1) (2) } \\
\hline & CG & 48 & $20-58$ & 2 months -4 years & 28 & (1) & \\
\hline \multirow{2}{*}{$\begin{array}{l}\text { Tian and Huang, } 2015 \\
\text { [29] }\end{array}$} & $\mathrm{EP}$ & 86 & $18-62$ & 8 weeks -5 years & 28 & $(3)+(4)$ & \multirow{2}{*}{ (1)(2)(3)(5) } \\
\hline & CG & 86 & $18-63$ & 9 weeks -7 years & 28 & (3) & \\
\hline \multirow{2}{*}{ Qin et al., 2016 [30] } & $\mathrm{EP}$ & 48 & $37.2 \pm 9.5$ & $1.0 \pm 0.4$ years & 28 & $(3)+(4)$ & (1)(2) (3) \\
\hline & CG & 48 & $36.7 \pm 8.6$ & $1.2 \pm 0.5$ years & 28 & (3) & (1)(2) \\
\hline Zhao, 2016 [31] & $\mathrm{EP}$ & 35 & $39.25 \pm 6.23$ & $4.49 \pm 2.26$ years & 30 & $(1)+(2)$ & (1) (2)(3)(4) \\
\hline $21100,2010[31]$ & CG & 35 & $38.28 \pm 7.23$ & $3.65 \pm 2.11$ years & 30 & (1) & \\
\hline
\end{tabular}

(1) Desloratadine: $5 \mathrm{mg}$ po qd; (2) Jade Screen: po tid + Desloratadine: $5 \mathrm{mg}$ po qd; (3) desloratadine: $8.8 \mathrm{mg}$ po qd; (4) Jade Screen: po tid + desloratadine: $8.8 \mathrm{mg}$ po qd; EP: experiment group; CG: control group; (1) total effective rate; (2) adverse reaction; (3) recurrence rate; (4) symptom score; (5) serum IgE level.

\section{Discussion}

Chronic urticaria is a clinically common skin disease that is hard to heal and often reoccurs even after it is suppressed; thus, it is worrisome to patients. The cause of this disease might be associated with food, drug, infection, inhalant, physical, genetic, endocrine, and mental factors [32]. However, it is difficult to identify the real etiological and predisposing factors in most patients, and relapse cannot be absolutely avoided even if a possible etiological factor is determined. The current treatment is mainly to use Western medicine to control the symptoms, but its effect is very limited. Thus, it is imperative to identify a novel therapeutic regime. With traditional Chinese medicine as the guideline, the present research found and verified a classic prescription with fewer side effects and a low reoccurrence rate, which could be a new method of treating chronic urticaria through the combination of Chinese traditional and Western medicines.

IgE is primarily generated from the plasma cells in the mucous membranes of the respiratory tract (nasopharynx, tonsils, and bronchi) and the digestive tract. It plays a crucial role in mediating the occurrence and development of allergic diseases [33]. Modern pharmacological research [34] suggests that by tonifying qi and strengthening exterior, Yupingfegn can boost immunity, improve allergic constitution, inhibit mastocyte release of bioactive substances, strengthen the $\mathrm{T}$ cell-mediated cellular immunity effect, increase the 


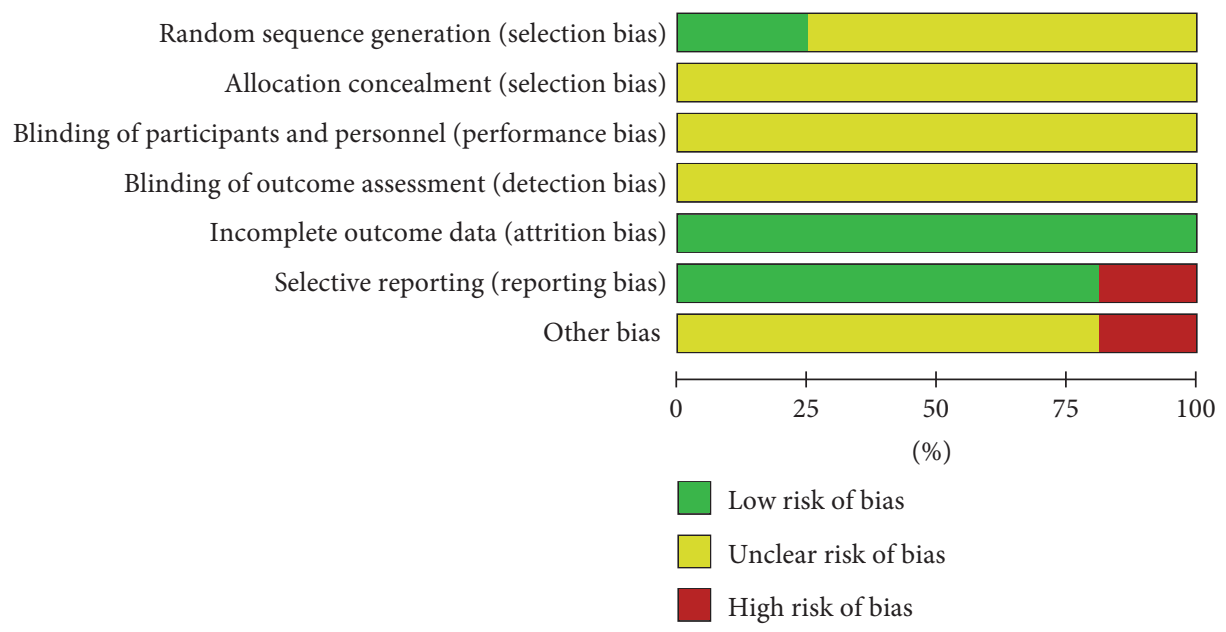

FIGURE 2: Risk of bias graph: review authors' judgments about each risk of bias item presented as percentages for all included studies.

number of T cells, and improve lymphocyte transformation rate [35]. Therefore, Jade Screen powder has been widely used to treat allergic skin diseases. Desloratadine has a long action time, a stronger antihistamine effect than other drugs of the same kind, a better antiallergic effect, selective cholinolytic activity, difficulty in passing through the blood-brain barrier, no central sedation and good patient compliance [36]; for these reasons, it has been widely used in the clinic as a first-line drug and a conventional antihistamine treatment. However, this drug only targets the disease process, so it cannot effectively control relapse. For the treatment of chronic urticaria, Jade Screen combined with desloratadine makes full use of Jade Screen to comprehensively strengthen the body's immunity, play the role of two-way regulation, improve IgA in the mucosal immune system, and act together with desloratadine to reduce IgE in hypersensitivity, regulate IgE-mediated humoral immunity, and significantly lower the IgE level of patients with urticaria [37].

In this research, we made a systematic evaluation on the functions of Jade Screen combined with desloratadine in treating chronic urticaria. Through a meta-analysis of the published studies, this research increased the sample size, enhanced the research credibility, and provided a reliable theoretical basis for clinical use.

First, the results of total effective rate and recurrence rate suggested that the total effective rate of the combination of traditional Chinese and Western medicine in treating chronic urticaria was higher than the single use of desloratadine, while the recurrence rate was lower than the single use of desloratadine.

Second, the results of occurrence rate of adverse reaction revealed that the combination of traditional Chinese and Western medicine and the single use of desloratadine showed no difference in the occurrence rate of adverse reactions. The adverse reactions of both treatments were mainly characterized by xerostomia, nausea, headache, dizziness, drowsiness, and, to a lesser extent, lack of strength.

Third, the results of the symptom score and total serum IgE showed high heterogeneity. The primary reason could be the small number of articles reviewed and their poor quality. Second, the published research literature about the curative effect of chronic urticaria in the clinic, the criteria for clinical symptom assessment, and intervention measures performance still cause much confusion, and no unified cure-judging criteria have been adopted, thus increasing the heterogeneity between the results of different research and having a high risk of selective bias.

The results of this study have satisfied our goal of this meta-analysis. The result has proven the effective therapeutic effect, fewer adverse reactions, and good safety of the traditional Chinese prescription Jade Screen in treating chronic urticaria. Thus, it can be used as an option for the treatment of chronic urticaria.

\section{Limitations and Advantages}

This research assesses the curative effect of Jade Screen in treating chronic urticaria according to the Methods for Cochrane Systematic Review for the first time. With limited cases included and low quality, the research fails to make a completely reliable positive conclusion. The limitations of the research included are as follows: (1) the research sites of all the articles were located in China, which is very likely to cause potential location bias; (2) all patients included were Chinese, and all the articles were written in Chinese or English, possibly resulting in potential selection bias and English language bias. (3) The articles included varied in dose, which may lead to potential drug dose bias. The advantages and deficiencies of the research were as follows. Deficiencies, (4) the methodological quality of the research included was low. All the research included mention "randomization", but only a few expound on how to generate a random sequence, and most research failed to describe how to realize allocation concealment. (5) The articles included have no unified diagnosis and efficacy determination criteria, increasing the heterogeneity between the results of different research and a high risk of selective bias. On the one hand, there is absence of the latest guidelines [38, 39] prepared 


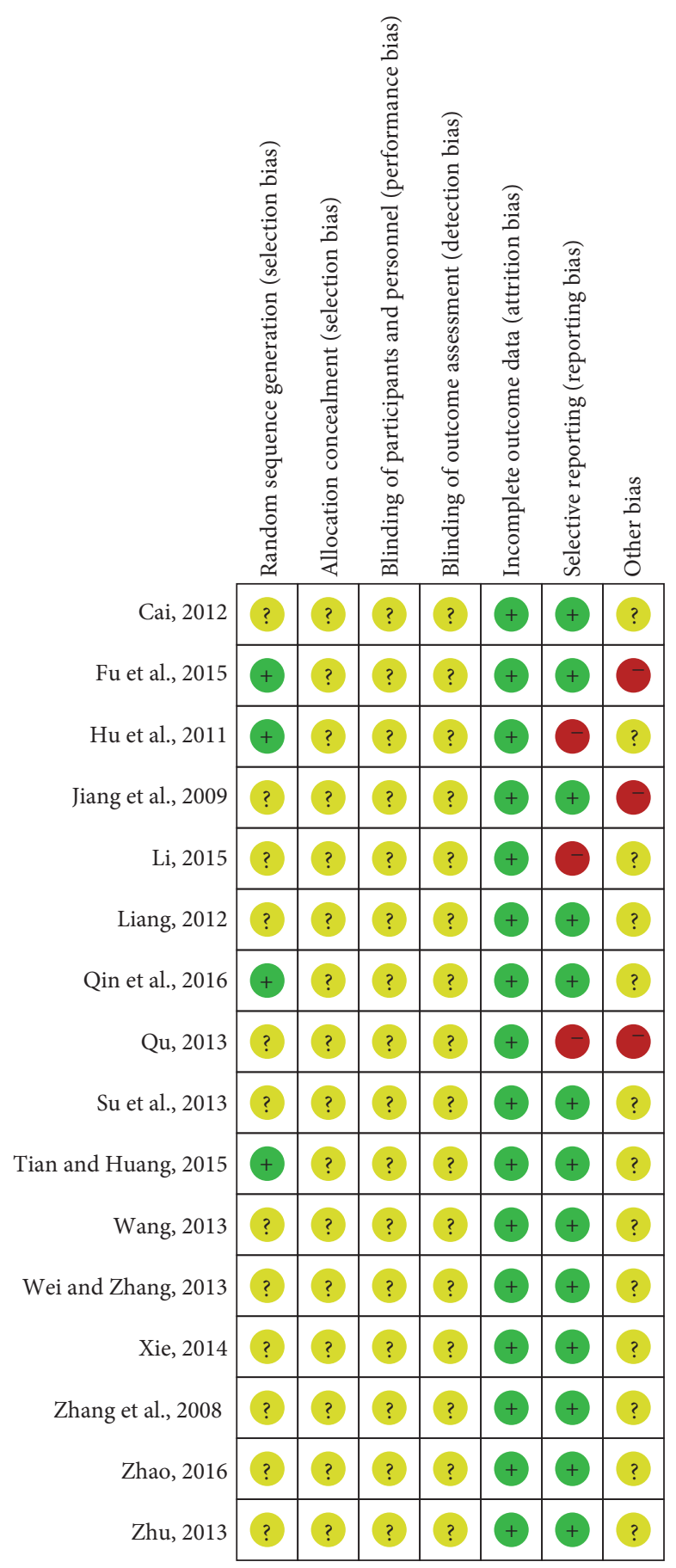

FIGURE 3: Risk of bias summary: review authors' judgments about each risk of bias item for each included study.

by evidence-based medicine methods in China; on the other hand, Chinese scholars probably do not pay much attention to it. Based on the above-mentioned bias factors, the efficacy and safety assessments of Jade Screen combined with desloratadine in treating chronic urticaria still require highquality clinical randomized controlled trials. It is necessary to conduct large sample, multicenter, high-quality clinical randomized controlled trials with long-term follow-up in the future.
With the development of examining approaches and the emphasis on patients' quality of life in recent years, many articles have included laboratory indices and quality of life into the clinical efficacy system of chronic urticaria [40]. For example, drug therapeutic effect and safety assessments are carried out based on changes in laboratory indices such as the Q-T segment in the ECG, blood routine tests, creatinine clearance rate, $\operatorname{IgE}$ and plasma histaminase before and after treatment $[41,42]$. With the furthering of traditional Chinese 


\begin{tabular}{|c|c|c|c|c|c|c|c|c|c|}
\hline Study or subgroup & \multicolumn{2}{|c|}{ Experimental } & \multicolumn{2}{|c|}{ Control } & Weight & $\begin{array}{c}\text { Odds ratio } \\
\text { M-H, fixed, } 95 \% \mathrm{CI}\end{array}$ & \multicolumn{2}{|c|}{$\begin{array}{c}\text { Odds ratio } \\
\text { M-H, fixed, } 95 \% \text { CI }\end{array}$} & \\
\hline Cai, 2012 & 50 & 62 & 32 & 58 & $8.0 \%$ & $3.39[1.50,7.65]$ & & $\longrightarrow$ & \\
\hline Fu et al., 2015 & 77 & 100 & 79 & 110 & $21.7 \%$ & $1.31[0.70,2.45]$ & & - & \\
\hline Hu et al., 2011 & 25 & 28 & 18 & 26 & $2.5 \%$ & $3.70[0.86,15.93]$ & & & \\
\hline Jiang et al., 2009 & 29 & 32 & 19 & 30 & $2.3 \%$ & $5.60[1.38,22.73]$ & & & \\
\hline Li, 2015 & 52 & 62 & 30 & 48 & $6.8 \%$ & $3.12[1.28,7.63]$ & & 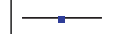 & \\
\hline Liang, 2012 & 58 & 60 & 50 & 60 & $2.1 \%$ & $5.80[1.21,27.73]$ & & & \\
\hline Qin et al., 2016 & 43 & 48 & 35 & 48 & $4.6 \%$ & $3.19[1.04,9.83]$ & & & \\
\hline $\mathrm{Qu}, 2013$ & 39 & 43 & 28 & 43 & $3.3 \%$ & $5.22[1.57,17.43]$ & & $\ldots$ & \\
\hline Su et al., 2013 & 50 & 59 & 43 & 58 & $8.3 \%$ & $1.94[0.77,4.87]$ & & $\square$ & \\
\hline Tian and Huang, 2015 & 80 & 86 & 66 & 86 & $5.8 \%$ & $4.04[1.53,10.65]$ & & $\longrightarrow$ & \\
\hline Wang, 2013 & 89 & 99 & 71 & 82 & $9.8 \%$ & $1.38[0.55,3.43]$ & & 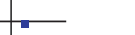 & \\
\hline Wei and Zhang, 2013 & 38 & 41 & 33 & 40 & $3.1 \%$ & $2.69[0.64,11.23]$ & & 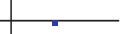 & \\
\hline Xie, 2014 & 45 & 60 & 30 & 60 & $9.4 \%$ & $3.00[1.38,6.50]$ & & 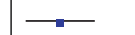 & \\
\hline Zhang et al., 2008 & 46 & 50 & 42 & 50 & $4.2 \%$ & $2.19[0.61,7.81]$ & & & \\
\hline Zhao, 2016 & 30 & 35 & 24 & 35 & $4.3 \%$ & $2.75[0.84,9.00]$ & & & \\
\hline Zhu, 2013 & 25 & 36 & 9 & 28 & $3.9 \%$ & $4.80[1.66,13.90]$ & & $\longrightarrow$ & \\
\hline Total (95\% CI) & & 901 & & 862 & $100.0 \%$ & $2.72[2.13,3.48]$ & & $\vartheta$ & \\
\hline Total events & 776 & & 609 & & & & & & \\
\hline \multicolumn{5}{|c|}{ Heterogeneity: $\chi^{2}=13.45, \mathrm{df}=15(P=0.57) ; I^{2}=0 \%$} & & 0.001 & 0.1 & 10 & 1000 \\
\hline \multicolumn{5}{|c|}{ Test for overall effect: $Z=7.97(P<0.00001)$} & \multicolumn{4}{|c|}{ Favours [experimental] } & \\
\hline
\end{tabular}

FIGURE 4: The meta-analysis forest map of the total effective rate of Jade Screen combined with desloratadine versus desloratadine alone for the treatment of chronic urticaria.

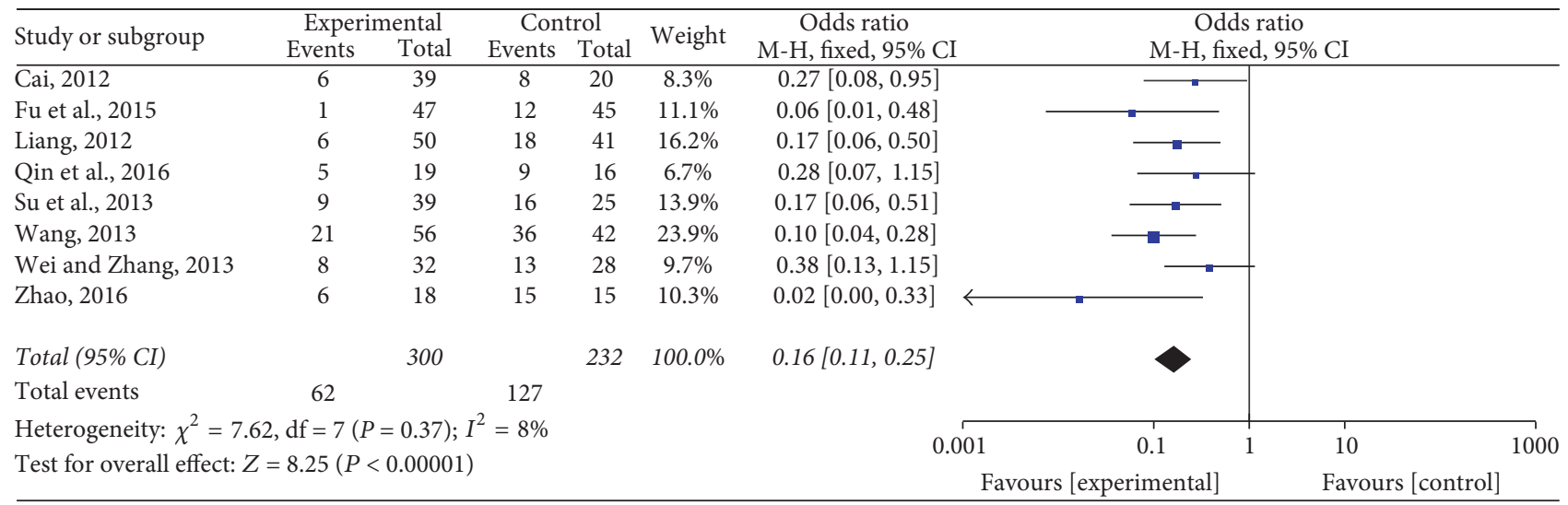

FIGURE 5: The meta-analysis forest map of the recurrence rate of Jade Screen combined with desloratadine versus desloratadine alone for the treatment of chronic urticaria.

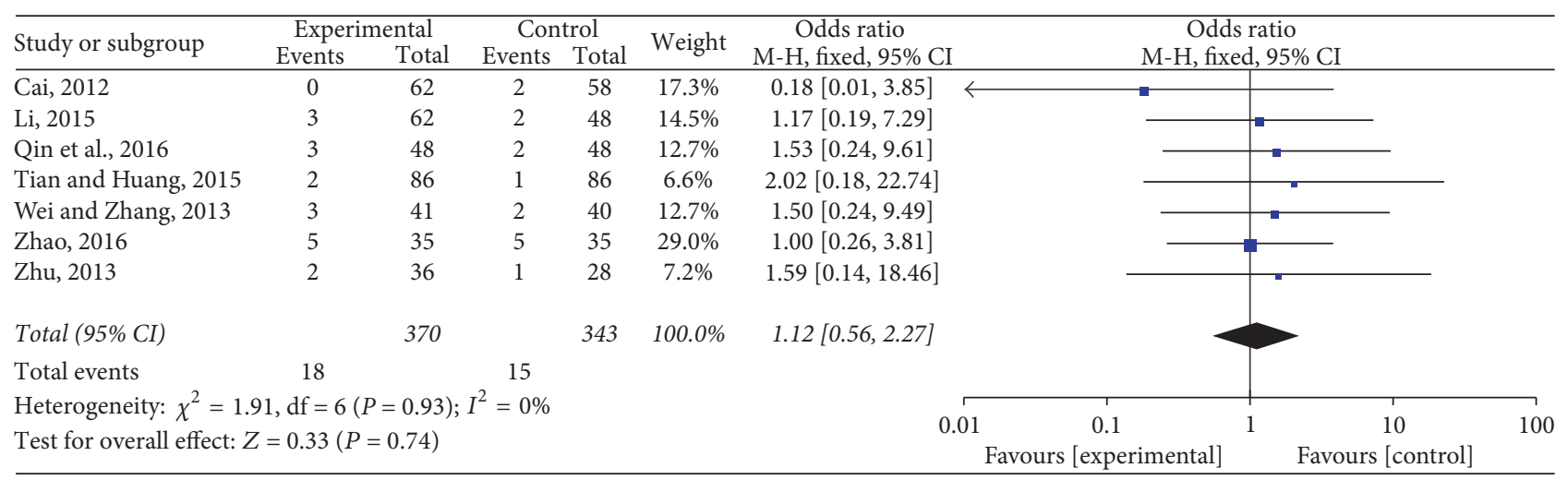

FIGURE 6: The meta-analysis forest map of the rate of adverse reaction of Jade Screen combined with desloratadine versus desloratadine alone for the treatment of chronic urticaria. 


\begin{tabular}{|c|c|c|c|c|c|c|c|c|c|c|c|}
\hline \multirow{3}{*}{$\begin{array}{l}\text { Study or subgroup } \\
\text { Cai, } 2012\end{array}$} & \multicolumn{3}{|c|}{ Experimental } & \multicolumn{3}{|c|}{ Control } & \multirow{2}{*}{ Weight } & \multirow{2}{*}{$\begin{array}{c}\text { Mean difference } \\
\text { IV, random, 95\% CI }\end{array}$} & \multirow{2}{*}{\multicolumn{3}{|c|}{$\begin{array}{c}\text { Mean difference } \\
\text { IV, random, 95\% CI }\end{array}$}} \\
\hline & Mean & $\mathrm{SD}$ & Total & Mean & $\mathrm{SD}$ & Total & & & & & \\
\hline & 1.29 & 1.752 & 62 & 2.33 & 2.45 & 58 & $12.6 \%$ & $-1.04[-1.81,-0.27]$ & \multicolumn{3}{|c|}{--1} \\
\hline Fu et al., 2015 & 0.34 & 0.26 & 100 & 0.68 & 0.16 & 110 & $49.6 \%$ & $-0.34[-0.40,-0.28]$ & 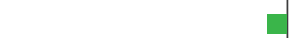 & & \\
\hline Jiang et al., 2009 & 0.3 & 3.2 & 32 & 3.1 & 5.3 & 30 & $2.0 \%$ & $-2.80[-5.00,-0.60]$ & & & \\
\hline Zhao, 2016 & 0.82 & 0.51 & 35 & 1.07 & 0.68 & 35 & $35.9 \%$ & $-0.25[-0.53,0.03]$ & 단 & & \\
\hline \multicolumn{3}{|l|}{ Total (95\% CI) } & 229 & & & 233 & $100.0 \%$ & $-0.44[-0.76,-0.13]$ & $\boldsymbol{\nabla}$ & & \\
\hline $\begin{array}{l}\text { Heterogeneity: } \tau^{2}= \\
\text { Test for overall effe }\end{array}$ & $\begin{array}{l}05 ; \chi^{2}= \\
Z=2.77\end{array}$ & $\begin{array}{l}=8.41, \\
(P=0\end{array}$ & $\begin{array}{l}\text {. }=3( \\
.006)\end{array}$ & $=0.04)$ & $I^{2}=$ & $64 \%$ & & & $\begin{array}{ccc}-4 & -2 & 0 \\
\text { Favours [experimental] }\end{array}$ & $\begin{array}{l}2 \\
\text { Favo }\end{array}$ & $\begin{array}{l}4 \\
\text { ours [control] }\end{array}$ \\
\hline
\end{tabular}

FIGURE 7: The meta-analysis forest map of the symptom scores of Jade Screen combined with desloratadine versus desloratadine alone for the treatment of chronic urticaria.

\begin{tabular}{|c|c|c|c|c|c|c|c|c|c|}
\hline \multirow{2}{*}{ Study or subgroup } & \multicolumn{3}{|c|}{ Experimental } & \multicolumn{3}{|c|}{ Control } & \multirow{2}{*}{ Weight } & \multirow{2}{*}{$\begin{array}{c}\text { Mean difference } \\
\text { IV, random, } 95 \% \text { CI }\end{array}$} & \multirow{2}{*}{$\begin{array}{c}\text { Mean difference } \\
\text { IV, random, 95\% CI }\end{array}$} \\
\hline & Mean & SD & Total & Mean & $\mathrm{SD}$ & Total & & & \\
\hline Fu et al., 2015 & 101.6 & 9.5 & 100 & 82.1 & 13.9 & 110 & $50.2 \%$ & $19.50[16.30,22.70]$ & \begin{tabular}{l|l} 
& $\square$
\end{tabular} \\
\hline Tian and Huang, 2015 & 88.62 & 25.12 & 86 & 120.21 & 24.67 & 86 & $49.8 \%$ & $-31.59[-39.03,-24.15]$ & [ \\
\hline Total (95\% CI) & & & 186 & & & 196 & $100.0 \%$ & $-5.93[-56.00,44.14]$ & \\
\hline $\begin{array}{l}\text { Heterogeneity: } \tau^{2}=12 \\
\text { Test for overall effect: }\end{array}$ & $\begin{array}{l}6.56 ; \chi \\
=0.23\end{array}$ & $\begin{array}{l}=152 \\
P=0.8\end{array}$ & $\begin{array}{l}88, \mathrm{df} \\
\text { 2) }\end{array}$ & $1(P<$ & 0.0000 & 1); $I^{2}$ & $=99 \%$ & & 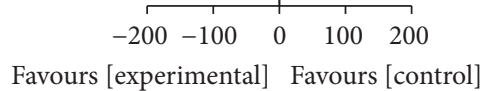 \\
\hline
\end{tabular}

FIGURE 8: The meta-analysis forest map of the serum total IgE level of Jade Screen combined with desloratadine versus desloratadine alone for the treatment of chronic urticaria.

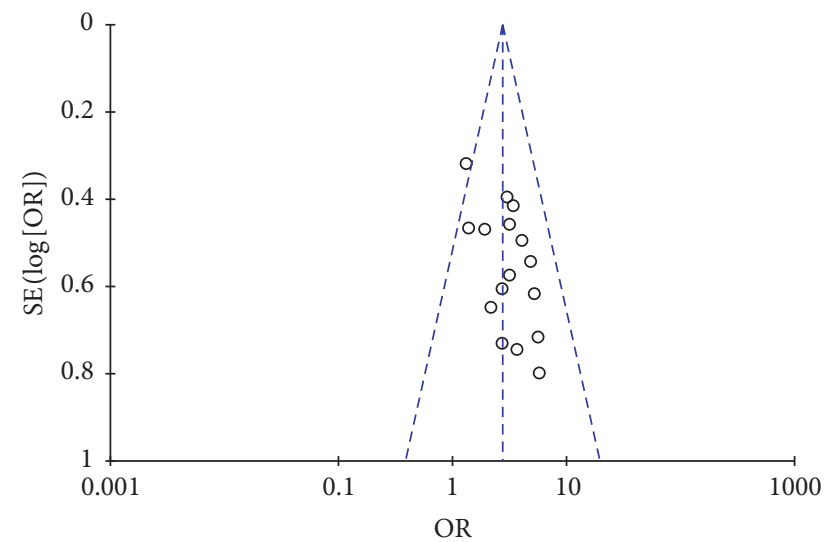

Figure 9: The funnel plot of the overall response rate of Jade Screen combined with desloratadine versus desloratadine alone for the treatment of chronic urticaria.

medicine in exploring the mechanism of chronic urticaria, there is an urgent need for China to establish an objective, effective, and extensive evaluation system that complies with the theory of TCM in order to better meet international standards. In future studies, we suggest using the Urticaria Activity Score (UAS) and quality of life to perform therapeutic evaluation and provide complete original data to further improve this meta-analysis in the future.

\section{Conclusion}

(1) The total effective rate of the combined use of Jade Screen and desloratadine was greater than that of desloratadine alone.
(2) The recurrence rate of the combined use of Jade Screen and desloratadine was greater than that of desloratadine alone.

(3) There was no difference in the incidence of adverse reactions between the two treatments.

(4) The present research results have showed that, in the treatment of chronic urticaria, the combination of traditional Chinese medicine and Western medicine is significantly better than using Western medicine alone. The therapeutic method provides a new thought for the treatment of chronic urticaria. However, due to the low quality of the currently selected articles, it is a necessity to carry out randomized controlled trials with strict designs in the future.

\section{Conflicts of Interest}

The authors declare that there are no conflicts of interest regarding the publication of this paper.

\section{Authors' Contributions}

Shao-fei Yuan and Jing-zhi Guan conceived the review and they contributed equally to this work as first authors. Shaofei Yuan performed literature searches electronically and Yong Hao performed manual literature searches. Feng-li Gao and Su-qin Shi performed study selection and data extraction. Jing-zhi Guan and Jian-yuan Chai performed quality assessment. Shao-fei Yuan and Jing-zhi Guan performed the analyses with Yong Hao, and Jian-yuan Chai, Feng-li Gao, Suqin Shi, Jian-xin Wang, and Jin He wrote the initial draft. Jihai Shi critically revised the paper. 


\section{Acknowledgments}

Thanks are due to Mrs. Cheng Cheng (China Mobile Group Inner Mongolia Company Limited) for downloading full text and using RevMan 5.3 software.

\section{References}

[1] T. Zuberbier, R. Asero, C. Bindslev-Jensen et al., "EAACI/ GA2LEN/EDF/WAO guideline: definition, classification and diagnosis of urticaria," Allergy, vol. 64, no. 10, pp. 1417-1426, 2009.

[2] M. Maurer, K. Weller, C. Bindslev-Jensen et al., "Unmet clinical needs in chronic spontaneous urticaria. A GA ${ }^{2}$ LEN task force report," Allergy, vol. 66, no. 3, pp. 317-330, 2011.

[3] B. F. O’Donnell, F. Lawlor, J. Simpson, M. Morgan, and M. W. Greaves, "The impact of chronic urticaria on the quality of life," British Journal of Dermatology, vol. 136, no. 2, pp. 197-201, 1997.

[4] M. Maurer, J.-P. Ortonne, and T. Zuberbier, "Chronic urticaria: an internet survey of health behaviours, symptom patterns and treatment needs in European adult patients," British Journal of Dermatology, vol. 160, no. 3, pp. 633-641, 2009.

[5] I. Baiardini, A. Giardini, M. Pasquali et al., "Quality of life and patients' satisfaction in chronic urticaria and respiratory allergy," Allergy: European Journal of Allergy and Clinical Immunology, vol. 58, no. 7, pp. 621-623, 2003.

[6] L. K. Delong, S. D. Culler, S. S. Saini, L. A. Beck, and S. C. Chen, "Annual direct and indirect health care costs of chronic idiopathic urticaria: a cost analysis of 50 nonimmunosuppressed patients," JAMA Dermatology, vol. 144, no. 1, pp. 35-39, 2008.

[7] T. Zuberbier, R. Asero, and C. Bindslev-Jensen, "EAACI/ GA(2)LEN/EDF/WAO guideline: management of urticaria," Allergy, vol. 64, no. 10, pp. 1427-1443, 2009.

[8] X. Yao, "Evaluation of the use strategy of antihistamines in chronic urticaria," American Journal of Dermatology and Venereology, vol. 37, no. 1, p. 16, 2015.

[9] F. Zeng, Y. Tang, and X. G. Liu, "Discussion about the dynamics of acupoints," Journal of Sichuan Traditional Chinese Medicine, vol. 26, no. 6, pp. 103-105, 2008.

[10] Chinese Pharmacopoeia Commission, Pharmacopoeia of People's Republic of China, Chemical Industry Publishing House, Beijing, China, 2005.

[11] W. Y. Zhu, Urticaria, Nanjing: Southeast University Publishing House, 2001, Southeast University Publishing House.

[12] Clinical guidelines of Chinese Medical Association, Fascicle of dermatology and Venereal Diseases, People's Medical Publishing House, Beijing, China, 2006.

[13] F. R. Liu, Practical dermatology, People's Medical Publishing House, Beijing, China, 3rf edition, 2004.

[14] B. Zhao, Clinical dermatology, Science and Technology Publishing House, Nanjing, China, 2001.

[15] X. J. Zhang, Dermatology and Venereology, People's Medical Publishing House, Beijing, China, 6th edition, 2004.

[16] B. J. Zhang, W. Q. Zhou, X. F. Zhang, and Z. G. Chen, "Effect of Jade Screen capsule combined with desloratadine on chronic urticaria," Practical Pharmacy and Clinical Remedies, vol. 11, no. 4, pp. 214-215, 2008.

[17] Y. Jiang, N. Ye, Y. C. Zhang, and Y. Zhou, "To observe the curative effect of Jade Screen particles combined with desloratadine tablets in the treatment of chronic urticaria," Journal of Guangdong Medical College, vol. 27, no. 1, pp. 58-59, 2009.
[18] B. Hu, J. Dai, and W. Li, "Effect of desloratadine combined with Jade Screen therapy on chronic urticaria and quality of life," Laboratory Medicine and Clinic, vol. 08, no. 14, pp. 1754-1755, 2011.

[19] H. Cai, "Therapeutic effect of Jade Screen dropping pills combined with western medicine on chronic urticaria," Psychologist magazine, vol. 4, no. 214, pp. 304-305, 2012.

[20] Q. S. Liang, "To observe the curative effect of desloratadine citrate disodium capsules joint Jade Screen particles in the treatment of chronic idiopathic urticaria," Chinese Journal of Clinical Rational Drug Use, vol. 5, no. 21, pp. 59-60, 2012.

[21] Y. H. Qu, "Clinical observation of desloratadine tablets joint Jade Screen particles in the treatment of chronic urticaria," Medical Aesthetics and Cosmetology, vol. 21, no. 1, pp. 90-91, 2013.

[22] S. N. Su, D. C. Yin, and X. H. Gu, "To observe the curative effect of desloratadine citrate disodium tablets joint Jade Screen dropping pills in the treatment of chronic urticaria," Medical information, vol. 26, no. 30, p. 228, 2013.

[23] J. Wang, "Clinical observation of desloratadine tablets combined with Jade Screen capsule in treatment of adult chronic urticaria," Strait Pharmaceutical Journal, vol. 25, no. 4, p. 241, 2013.

[24] X. Wei and H. B. Zhang, "Clinical observation of desloratadine citrate disodium tablets combined with Jade Screen capsule in treatment of 81 cases of chronic urticaria," Chinese Journal of Dermatovenereology of Integrated Traditional and Western Medicine, vol. 12, no. 4, pp. 249-250, 2013.

[25] X. L. Zhu, "Clinical observation of Jade Screen capsule combined with desloratadine in treatment of chronic urticaria," China Healthcare \& Nutrition, vol. 23, no. 6, p. 3216, 2013.

[26] L. H. Xie, "Clinical observation of Jade Screen grain combined with desloratadine dispersible tablets in treatment of chronic urticaria," Journal of Aerospace Medicine, vol. 25, no. 6, pp. 825826, 2014.

[27] H. D. Fu, Z. Zhang, and M. Xu, "Curative effect observation of Jade Screen grain adjuvant therapy of blood deficiency and wind dryness type chronic urticaria," Journal of New Chinese Medicine, vol. 47, no. 7, pp. 174-176, 2015.

[28] L. F. Li, "Efficacy of desloratadine combined with Jade Screen in the treatment of chronic urticaria," Journal of Practical Medical Techniques, vol. 22, no. 3, pp. 299-300, 2015.

[29] J. Tian and S. B. Huang, "Therapeutic observation of the effect of combining therapy of desloratadine citrate disodium capsules and yupengfeng on chronic urticaria and effects on serum levels of IgE," Chinese Journal of Medicine, vol. 50, no. 3, pp. 79-81, 2015.

[30] F. Qin, H. X. Que, and C. J. Cui, "Clinical curative effect observation of Jade Screen capsule combined with desloratadine tablets in treatment of chronic urticaria," The Journal of Medical Theory and Practice, vol. 29, no. 5, pp. 639-640, 2016.

[31] P. Zhao, "Clinical observation of desloratadine combined with Jade Screen capsule in the treatment of chronic idiopathic urticaria," World Chinese Medicine, vol. 11, no. B06, pp. 17701771, 2016.

[32] Z. Y. Li, J. F. Wang, and H. Y. Zhang, "Research Advances in Chronic Urticaria with Helicobacter pylori Infection," Clinical Journal of Traditional Chinese Medicine, vol. 27, no. 12, pp. 17891791, 2015.

[33] Q. H. Huang, Y. X. Lv, J. M. Zhou, and L. J. Zhen, "Clinical value of $\operatorname{IgE}$ and eosinophils in children with acute and chronic 
urticaria," Journal of Mathematical Medicine, vol. 28, no. 11, pp. 1630-1631, 2015.

[34] Y. L. M. Mu, "Study on pharmacological characteristics of Chinese prescription Jade Screen," International Journal of Traditional Chinese Medicine, vol. 28, no. 2, pp. 96-97, 2006.

[35] H. T. Xin, J. L. Hou, S. H. Xu, and X. H. Zhou, "Progress in the study of immune pharmacology of Jade Screen powder," China Journal of Chinese Materia Medica, vol. 23, no. 8, pp. 505-507, 1998.

[36] R. S. Geha and E. O. Meltzer, "Desloratadine: A new, nonsedating, oral antihistamine," The Journal of Allergy and Clinical Immunology, vol. 107, no. 4, pp. 751-762, 2001.

[37] H. Chen, "Therapeutic observation of the effect of yupengfeng powder on chronic urticaria and effects on serum levels of IgE," Journal of Chinese Medicinal Materials, vol. 26, no. 2, pp. 151-152, 2003.

[38] Z. G. Zhang, X. H. Wang, and Q. N. Zhang, "Potential Bias Due to the Defi nition of RCTs for Inclusive Criteria of Systematic Reviews Chinese Journals," Chinese Journal of Evidence-Based Medicine, vol. 13, no. 6, pp. 764-767, 2013.

[39] J. T. Liu, C. Y. Qin, and H. Dang, "Interval Estimation for the Amount of Heterogeneity in Meta-Analysis Based on QStatistic Following Linear Transformation of Chi-Square Distribution," Chinese Journal of Evidence-Based Medicine, vol. 12, no. 11, pp. 1404-1406, 2012.

[40] Y. P. Ma, Investigation on quality of life and clinical features of chronic urticaria and Study on the correlation of basophils in chronic idiopathic urticaria, Beijing: Beijing Union Medical College, Beijing Union Medical College, Beijing, China, 2010.

[41] J. J. Liu, "Effects of anti histamine drugs combined with placenta polypeptide on cellular immunity in patients with chronic urticaria," Hebei Medical Journal, vol. 32, no. 9, pp. 1110-1110, 2010.

[42] D. Y. Wang and B. Liu, "Clinical observation of cetirizine hydrochloride in the treatment of urticaria," The Chinese Journal of Dermatovenereology, vol. 3, no. 2, p. 143, 2001. 


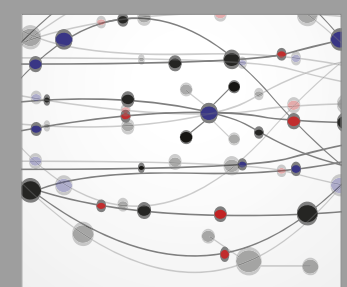

The Scientific World Journal
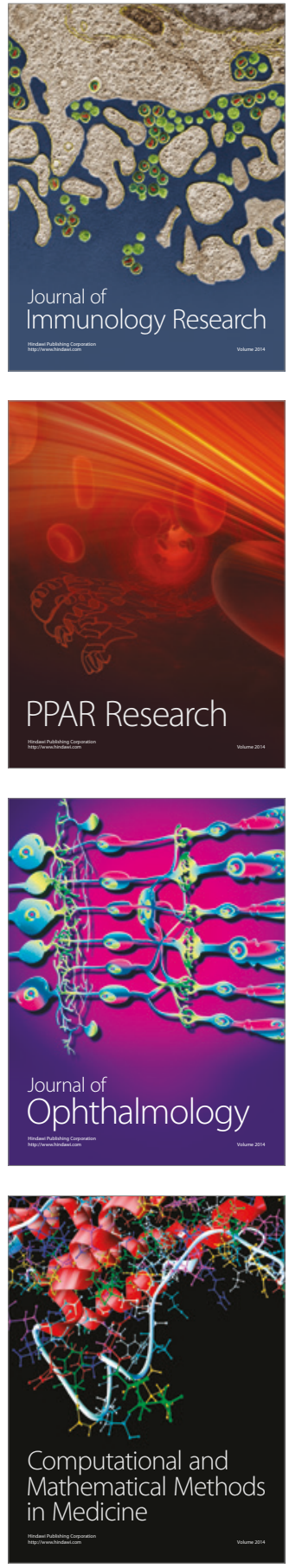

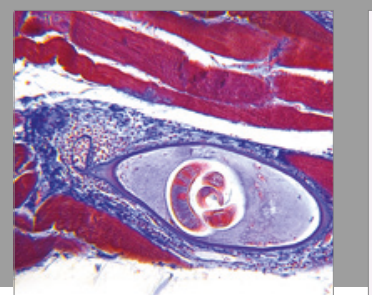

Gastroenterology Research and Practice
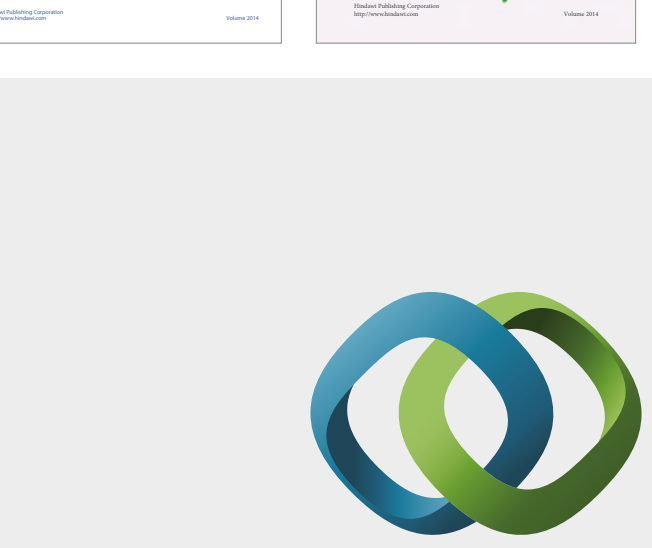

\section{Hindawi}

Submit your manuscripts at

https://www.hindawi.com
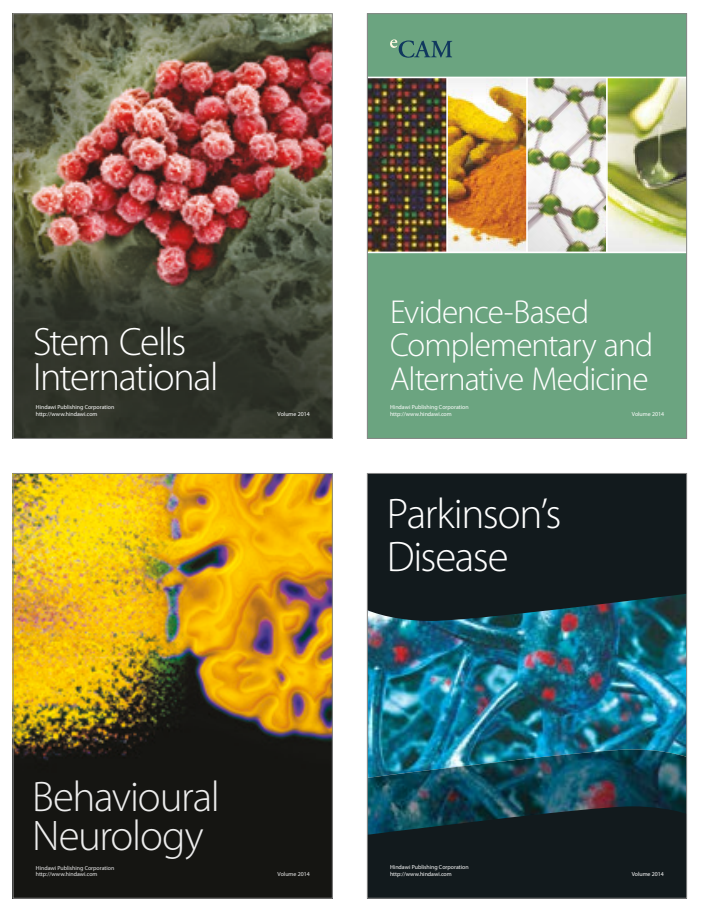
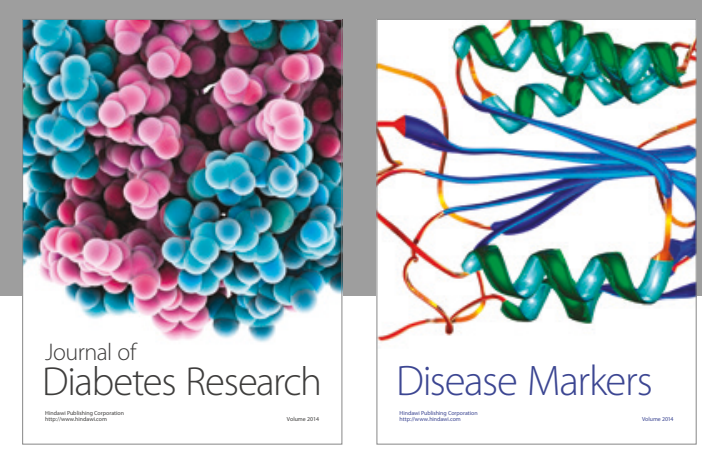

Disease Markers
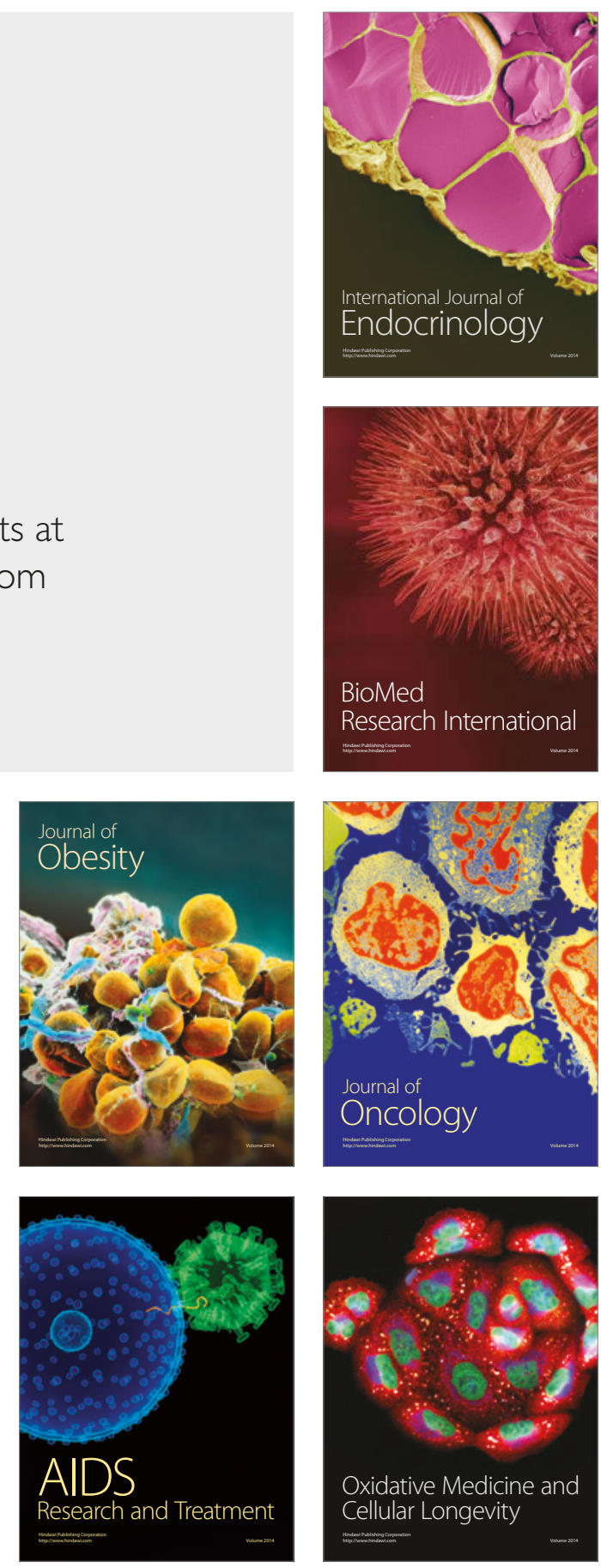